\title{
Phase and Atomic Displacement Profiles within Crystals Measured and Simulated using the Self-interference of Split HOLZ Lines
}

\author{
Mana Norouzpour and Rodney Herring
}

CAMTEC, Mechanical Engineering, University of Victoria, Victoria, Canada V8W 2Y2

Measuring atomic displacement inside crystals has been an important field of interest for decades especially in the semiconductor industry for its effect on the electron/hole mobility and necessary for the development of quantum computers based on strain bandgap engineering. Among all available transmission electron microscopy (TEM) methods offering nano-scale resolution measurements, convergent beam electron diffraction (CBED) shows the highest sensitivity to the atomic displacement. CBED's higher order Laue zone (HOLZ) lines split by small variations of the atomic lattice making it possible to measure the atomic displacement through the crystal. However, it is a cumbersome measurement and it could only reveal the atomic displacement in two dimensions, i.e., within the $x, y$ plane of the thin film TEM specimen. The z-axis atomic displacement through the specimen thickness along the path of the electron beam has been missing. This information can now be obtained by recovering the phase information across the HOLZ line that splits due to the atomic displacement using the self-interference of the split HOLZ line (SIS-HOLZ) as recently reported [1,2,3]. The analytical method used to simulate the phase profile across the split HOLZ line is presented here for the first time. The kinematical theory of diffraction using Howie-Whelan equation is used. It is acceptable for this study as the specimen thickness is smaller than the extinction distance, $\varepsilon_{\mathrm{g}} / \pi$, of the diffraction vector, $g$ of the split HOLZ line [4]. Thus, the SIS-HOLZ method determined the atomic displacement through the crystal by applying the Howie-Whelan equation to obtain the phase, $\Phi(z)$, across the split HOLZ line using $\tan ^{-1} \operatorname{Im}(\Phi(\mathrm{z})) / \operatorname{Re}(\Phi(\mathrm{z}))$.

In this work the phase profile simulations are implemented for the 008 split HOLZ line assuming three different atomic displacement profiles of V-shape, cosine bell-shape and cosine pseudo- parabola through the specimen thickness (Fig. 1a-1b). In order to satisfy the kinematical theory of diffraction the specimen thickness must be smaller than $171 \mathrm{~nm}$ considering $\xi_{008(\mathrm{Si})}=540 \mathrm{~nm}$. Images of the experimental 008 split HOLZ line were taken by means of Hitachi's HV-3300v STEHM operated at 200 $\mathrm{kV}$ within the $\mathrm{Si}$ substrate at different distances from the interface of the $\mathrm{Si} / \mathrm{Si}_{0.8} \mathrm{Ge}_{0.2}$ superlattice. The split HOLZ line at $80 \mathrm{~nm}$ away from the interface is good in terms of intensity and split width to selfinterfere (Fig. 2a-2c). The voltage of $4.5 \mathrm{~V}$ was applied to the centrally aligned biprism in the split (Fig. 2d) to obtain the interference fringes within the interferogram (Fig. 2e). Using the holoworks subroutine of DigitalMicrograph helped with the phase image and profile reconstruction (Fig. $2 \mathrm{f}-2 \mathrm{~g}$ ). The experimental phase profile shows a good fit with the simulated cosine bell-shape atomic displacement profile (Fig. 2h-2i). Additionally, an atomic displacement amplitude of $\mathrm{R}=0.0325 \mathrm{~nm}$ at the center of the specimen (Fig. 2i) was determined using SIS_HOLZ whereas it is 0.0294 using only the width of the split HOLZ, i.e., the previous capability. SIS-HOLZ method makes possible the determination of the full 3D strain existing within crystals necessary for many types of science studies.

[1] M. Norouzpour et al. Journal of Materials Research (2017), p.1-13

[2] M. Norouzpour et al. M\&M (2015), p. 1965-1966.

[3] R. Herring et al. Ultramicroscopy, 156 (2015), p. 37-40.

[4] C. Alfonso et al. Ultramicroscopy, 110 (2010), p. 285-296. 

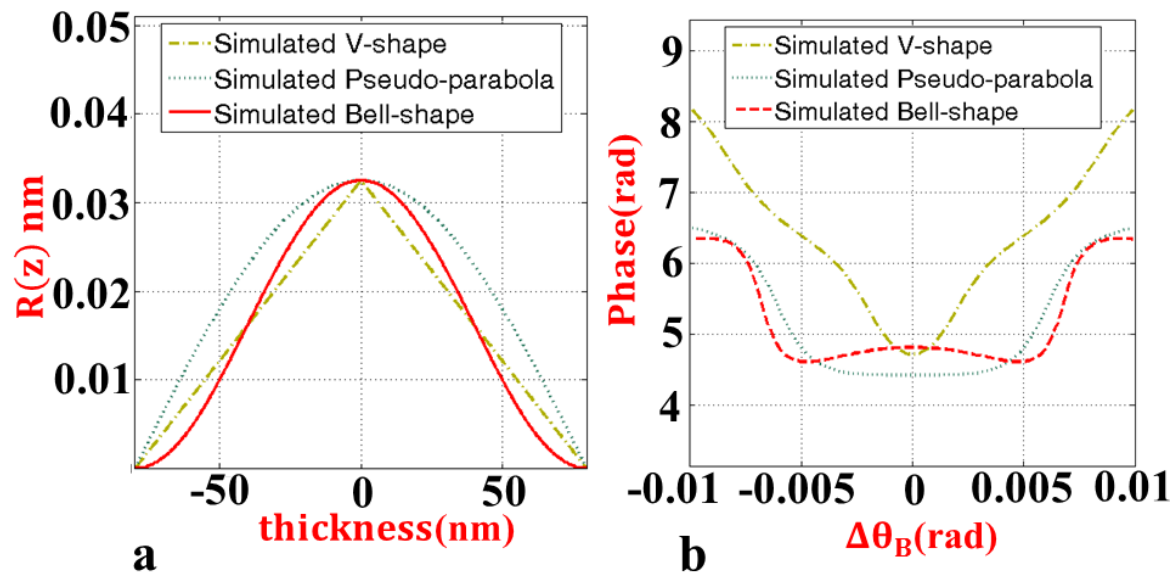

Fig. 1- (a) the displacement profiles through the thickness of the crystal, $t=160 \mathrm{~nm}$, (b) the simulated phase profiles for the corresponding displacement profiles of the 008 split HOLZ line.

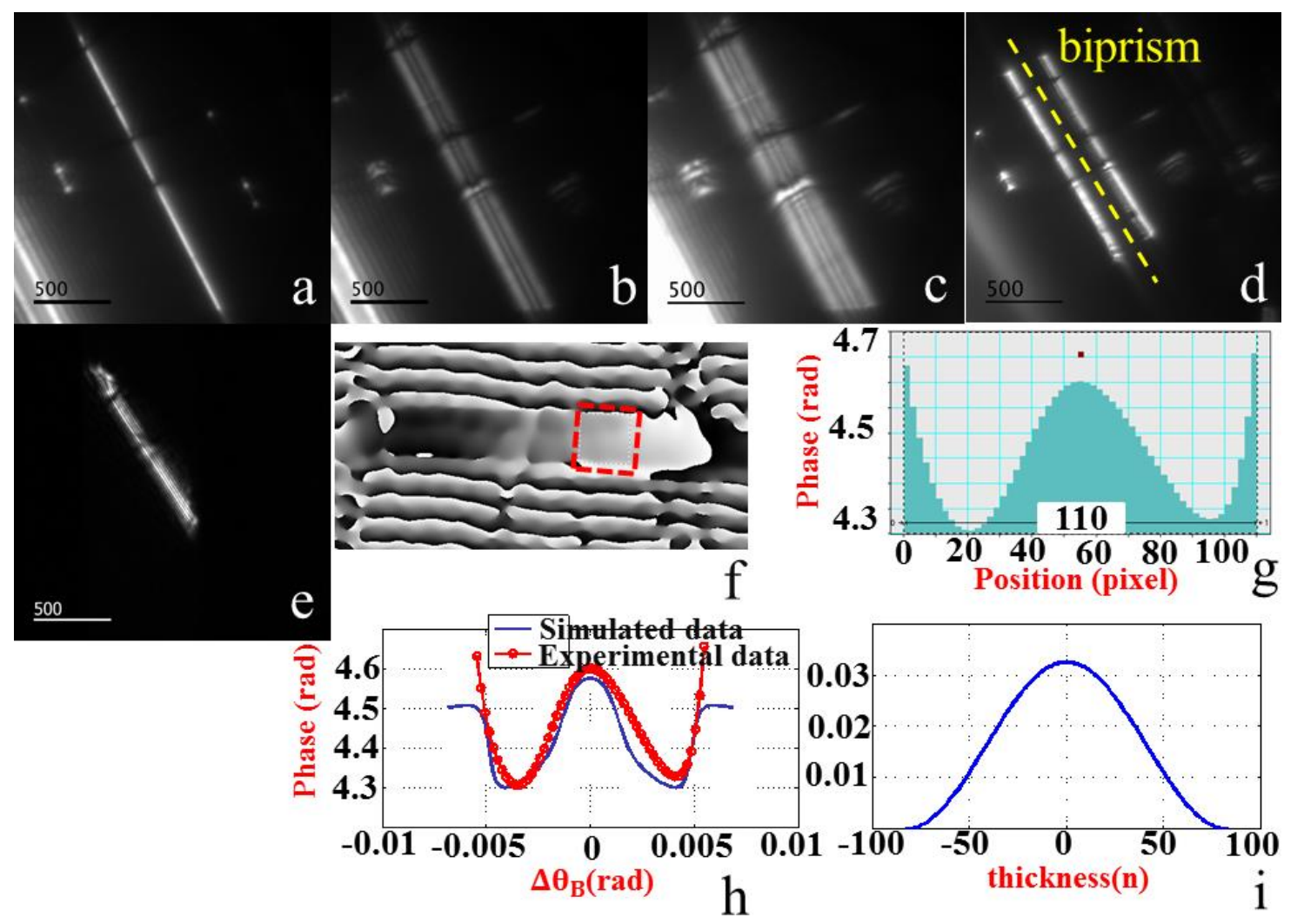

Fig 2 - Experimental images of the dark field disk of the 008 split HOLZ line obtained away from the $\mathrm{Si} / \mathrm{Si}_{0.8} \mathrm{Ge}_{0.2}$ interface within $\mathrm{Si}$ at (a) $400 \pm 10 \mathrm{~nm}$, (b) $100 \pm 10 \mathrm{~nm}$, (c) $80 \pm 10 \mathrm{~nm}$, (d) the aligned biprism with a negative potential centered within the split HOLZ line, (e) the interferogram of the selfinterfered split HOLZ line showing fine fringes running parallel with the length of the HOLZ line, as well as, the intersections of other HOLZ lines (f) the reconstructed phase image of (e) oriented horizontally, (g) the phase profile passing through its width across the sketched red box within the phase image, (h) the calculated cosine bell-shape phase profile fits the experimental phase profile, (c) The reconstructed atomic displacement profile at $80 \mathrm{~nm}$ distance from the $\mathrm{Si} / \mathrm{Si}_{0.8} \mathrm{Ge}_{0.2}$ interface along the $\mathrm{z}-$ axis through the thickness of the crystal specimen. 